\title{
The Deceptive Manifestations of Treatment Resistance in Depression: A New Look at the Problem
}

\author{
Giovanni A. Fava ${ }^{a}$ Fiammetta Cosci $^{b}$ Jenny Guidic Chiara Rafanellic \\ ${ }^{a}$ Department of Psychiatry, University at Buffalo, State University of New York, Buffalo, NY, USA; ${ }^{b}$ Department of \\ Health Sciences, University of Florence, Florence, Italy; ' ${ }^{\top}$ Department of Psychology, University of Bologna, \\ Bologna, Italy
}

In the past decade, the number of papers dealing with treatment resistance in depression has increased to a considerable degree. It has recently been discussed whether current definitions of treatment resistance are appropriate: issues of misdiagnosis, diagnostic and biological heterogeneity, and suboptimal therapeutic strategies have been raised [1-3]. However, current conceptualizations of treatment resistance focus on the characteristics of the patient (whether neurobiological assets, or attitudes, or psychiatric comorbidity) for the insufficient effectiveness of antidepressant drugs and omit any reference to the potential iatrogenic effects of treatment [4], as if, in the field of infectious disease, treatment resistance were conceptualized independently of the previous use of antibiotics.

We will try to outline some historical developments of the concept of treatment resistance in depression and how a different assessment strategy is required to address current clinical challenges.

\section{The Development of the Concept}

Definitions of treatment resistance in depression are generally based on failure to respond to a trial with antidepressant drugs or, with a more stringent specification, on insufficient responses to at least two courses of adequate treatment [5]. Adequate drug treatment is defined as use of antidepressant drugs at doses significantly superior to placebo in double-blind studies administered continuously for a minimum duration of 6 weeks [5]. Simpson and Kessel [6] provided an important specification: one trial should include the use of a high-dose (200$300 \mathrm{mg}$ ) tricyclic antidepressant such as imipramine for a minimum of 6 weeks. Such a specification was justified by the apparent superiority of tricyclics over other antidepressants in severe depression and their dose-response characteristics [7], despite the fact that a recent metaanalysis of trials comparing tricyclics to selective serotonin reuptake inhibitors failed to show any significant difference in efficacy for the average patient [8]. More recently, with the replacement of tricyclics by second generation antidepressants, switching and augmentation strategies have been advocated, despite their overall lack of efficacy $[9,10]$. The basic clinical concept is the fact of using the term resistance only when the best therapeutic options had been used, not on a simple trial basis. For instance, in one of the early trials on the use of psychotherapeutic strategies in treatment-resistant depression [11], the definition of Simpson and Kessel [6] was used before applying cognitive-behavioral strategies to the pa- karger@karger.com

(c) 2020 S. Karger AG, Basel

www.karger.com/pps

Karger"
Chiara Rafanelli, MD, $\mathrm{PhD}$

Department of Psychology, University of Bologna Viale Berti Pichat 5

IT-40127 Bologna (Italy)

chiara.rafanelli@unibo.it 
tient population. Further, a 2-year follow-up was available. The authors concluded that a trial of cognitive-behavioral therapy (CBT) by an experienced therapist should be performed before labeling an episode of major depression as "refractory" or "treatment resistant" [11]. These latter terms should apply only when a psychotherapeutic effort has been made. Until then, it seems more appropriate to define depression as "drug refractory" or "drug resistant."

Other important aspects that emerged were the difficulties in determining a simple categorical distinction between responses and nonresponses in depression. Following the introduction of the concept of staging in psychiatry in 1993 [12], different methods to stage the degree of treatment resistance in patients with unipolar depression have been suggested $[3,13]$. There is no consensus about the superiority of a single staging method. Thase and Rush [14] proposed a 5-stage model: patients were classified according to the number and classes of antidepressants that failed to produce a response, with staging moving from more common to less common treatments. Thus, for instance, stage 1 was characterized by failure of at least one adequate trial of one major class of antidepressant. According to a second model, called European approach [15], stage A represented no response to one adequate antidepressant trial lasting 6-8 weeks; treatment-refractory depression, stage $B$, was the failure to 2 or more adequate trials of different antidepressants given in adequate dosages for a period of at least 12-16 weeks but no longer than 1 year; chronic resistant depression (stage C) was due to resistance to several antidepressant trials, including augmentation strategy, lasting 12 months or more. The Massachusetts General Hospital model considered both the number of failed trials and the intensity or optimization of each trial without assumptions on the hierarchy of antidepressant classes. This method generates a continuous variable reflecting the degree of resistance in depression and ranging from 0 to 5 [5].

Finally, the Maudsley Staging Method incorporated, in addition to the number of failed treatment trials, factors considered to be closely related to the depressive illness itself: duration, severity, treatment failure to antidepressants, use of augmentation, and use of electroconvulsive therapy [16]. At the end, the stage of treatment resistance was represented as a single score ranging from 3 to 15 . An attempt to integrate the various models including the use of psychotherapeutic strategies was finally suggested [13]. The basic principle is that it is quite different to treat patients with a major depressive episode who displayed a positive response to previous therapeutic trials (stage 0) and patients who failed to respond to at least three adequate trials (including psychotherapy) of which at least one concerned augmentation/combination or electroconvulsive therapy (stage 4).

\section{The Missing Dimensions}

A first problem, which was acknowledged by many investigators, concerned the difficulties in drawing a separation line in the Hamilton Depression Rating Scale scores [17] among nonresponse ( $<25 \%$ symptom reduction from baseline), partial response (25-49\% symptom reduction), and response without remission $(50 \%$ or greater reduction) [18]. In this latter case, the evaluation of the presence of residual symptomatology that interferes with quality of life and functioning despite improvement of depressed mood [19] may blur the distinction between resistant and nonresistant depression. Yet, what is neglected in this approach is the difference between insufficient or no benefits and clinical worsening. Re-analyzing the Beta-Blocker Heart Attack Trial, Horwitz et al. [20] found that propranolol reduced the risk of dying for the "average" patient who survived an acute myocardial infarction, whereas it was harmful in a subgroup of patients characterized by specific co-therapy histories. If we accept the possibility that a treatment which is helpful on average may be ineffective on some and even harmful on someone else, we may identify a subgroup of patients who deteriorate upon treatment. In the methodological recommendations for trials in psychological interventions [21], it is suggested to indicate in a trial the number of patients who deteriorate, who would otherwise be masked by the definition of failure to improve. And these cases indeed occur in trials [20] as well as in practice [22].

A second neglected aspect has to do with the issue of responsiveness. Richardson and Doster [23] suggested the importance of considering three dimensions in the process of evidence-based decision: baseline risk of poor outcomes from an index disorder without treatment (benefits), responsiveness to the treatment option, and vulnerability to the adverse effects of treatment. Labeling what falls outside the limits of responsiveness as resistance is questionable. For instance, anxious depression is less likely to respond to antidepressant drugs compared to non-anxious depression [24]. In a sample characterized by anxiety and depression of mild severity we can shift into the realm of resistance what is simply the result of a treatment that has limited efficacy. Similar consider- 
ations may apply to the limited effectiveness of novel antidepressants [25].

A rational approach to treatment takes into account the balance between potential benefits and adverse effects applied to the individual patient [23]. Yet, the iatrogenic effects of treatment are carefully avoided in the literature on treatment resistance in depression [4]. This is a major omission, as the following examples indicate. A particular form of resistance occurs when a drug which resulted in clinical response in previous episodes is no longer effective when it is started again after a drug-free period. The prevalence of this type of resistance varies but may occur in up to one third of patients $[26,27]$. Resistance may also ensue with loss of clinical effect in a patient who previously responded to antidepressant drug treatment. The return of depressive symptoms during maintenance antidepressant treatment was found to occur in a significant proportion of patients in published trials $[26,28]$. Increase in dosage may only yield temporary improvement [26]. Use of antidepressants may be associated not only with the return of depressive symptoms during maintenance treatment (loss of clinical effect), but also with the appearance of new symptoms and exacerbation of baseline clinical picture (paradoxical effects). Improvement may result from antidepressant discontinuation [26]. Finally, before entering a trial, patients may discontinue their current antidepressant treatment. Even with slow tapering, the likelihood of withdrawal syndromes and persistent postwithdrawal disorders and symptoms is very high and the likelihood of response of these syndromes to the introduction of new antidepressants is very variable [29-33].

Resistance to re-challenge, loss of clinical effects, paradoxical reactions, withdrawal and postwithdrawal syndromes tend to cluster and may share a common mechanism that has been subsumed under the rubric of oppositional model of tolerance $[26,32]$. Very seldom these phenomena are considered in a trial. An exception is the ADAPT trial that excluded patients who presented with loss of therapeutic response. The trial failed to detect differences between low-dose aripiprazole and placebo in treatment-resistant depression despite a good sample size, a sophisticated design, and a careful assessment [34]. Further, short-term effects may be related to a phenomenon (e.g., withdrawal) that is not considered from the beginning and may wane with duration of treatment. In other words, depending on the type of sample, a drug may be more effective than placebo in overcoming withdrawal and postwithdrawal symptomatology and not necessarily depressive symptoms.

Treatment Resistance

\section{Flawed Designs and Unwarranted Assumptions}

In clinical practice, adapting the treatment plan to the changing status of the individual patients is a common strategy. It takes into account both the history of previous treatments and the response to those treatments. When Sir Austin Bradford Hill transferred the methodology that had accumulated with randomized trials in agricultural sciences in the first double-blind placebo-controlled study in medicine [35], the scenario was dominated by the challenges that acute diseases entailed. Tuberculosis was widespread, there were many reports in the literature claiming effectiveness of drug therapy, and the amount of streptomycin that was available was limited [36]. Randomized controlled trials (RCT) in medicine thus referred to an average patient who fulfilled the criteria for admission and ignored the patient's individual history [37]. There were good reasons for the choices: most clinical encounters were for acute diseases, and patients were unlikely to have experienced many treatments before (such as repeated exposure to antibiotics). Today most of the clinical activities are concentrated on chronic disease or non-disease-specific complaints [38]. Yet, the standard randomized controlled trial design is still based on the acute disease model and ideally evaluates therapeutic effects in untreated patients who have a recent acute onset of their disturbances. This is in sharp contrast with the fact that previous treatments may have actually modified the course and responsiveness of the individual patient $[18,38]$.

Adaptive interventions [39] or adaptive treatment strategies [38] have been developed for adjusting a treatment plan to the changing state of an individual patient. An adaptive intervention is a multistage process that is based on patients' characteristics and intermediate outcomes collected during an intervention, such as the patient's response after the first line of treatment [39]. The STAR*D trial [40] was based on adaptive interventions that followed non-response to initial treatment, such as switching to or augmentation with another type of treatment, potentially modifying treatment response. The aim of the trial was to apply the best pharmacological strategies for obtaining remission in major depression using the least conservative definition of treatment-resistant depression (one failed trial with citalopram). Those who did not recover were submitted to four sequential steps involving switching, augmentation, and combination strategies based on available literature. The results were rather disappointing. The cumulative rate of remission after 4 sequential steps was $67 \%$ and, when sustained re-

Psychother Psychosom 2020;89:265-273 DOI: $10.1159 / 000507227$ 
covery (taking into account relapse rates while under treatment) was considered, the cumulative rate was $43 \%$. This means that the strenuous efforts after step 1 (open treatment with citalopram) yielded an additional $6 \%$ of sustained recovery. Even though each step of the trial was carefully conceived to increase the likelihood of response in patients who did not remit, remission rates decreased after each treatment step [40]. Rates of relapse increased after each treatment step in patients who achieved remission. Further, intolerance (dropouts for any reason during the first 4 weeks or side effects afterwards) increased after each treatment step. Many of the STAR*D findings can be interpreted in light of the oppositional model of tolerance [32]: pharmacological manipulations, either by switching or augmentation (steps 1 and 2) may propel depressive illness into a refractory phase, characterized by low remission, high relapse, and high intolerance (steps 3 and 4).

The conceptual assumption of the STAR*D and other trials concerned with treatment-resistant depression is that, after testing a standard treatment in a group of patients, we are left with a fairly homogeneous group characterized by resistance. The basic flaw is to assume improvement to be a unitary phenomenon [41]. Actually, non-response includes a very wide range of explanations (inadequate treatment in terms of duration, doses or indications; the occurrence of side effects prevailing over benefits; incomplete adherence; previous exposure to that specific treatment; psychosocial events intervening during the trial; problems in the patient-doctor relationship; modifications in patient's lifestyle and illness behavior) [42]. In particular, manifestations of tolerance induced by previous treatments (e.g., loss of clinical effects, withdrawal syndromes) may affect the results [4]. The term "iatrogenic comorbidity" refers to the unfavorable modifications in the course, characteristics, and responsiveness to treatment of an illness that may be related to previously administered therapies $[4,43]$. Such vulnerabilities may manifest themselves during treatment administration and/or after its discontinuation. The changes are persistent and not limited to a short phase such as in the case of persistent postwithdrawal disorders [4]. Timing may add heterogeneous features: certain individuals may change readily with respect to certain symptoms, whereas other subjects may have opposite tendencies [41]. As a result, designs that use highly heterogeneous populations at the start and are based on identification of non-response end up following an oversimplified conceptual model that clashes with the clinical reality they would attempt to mimic.

\section{The Wreck of Depression Management}

The conceptual flaws and the spurious results of the literature have yielded two detrimental clinical consequences. One is the development of drugs geared to treatment resistance, such as ketamine/esketamine [25], which would not survive the test of a classic RCT in depression. The second consequence has been the assumption that treatment with whatever antidepressant is right in the first place, and failure to respond is entirely shifted (and implicitly blamed) upon patients' characteristics. Treatment resistance thus calls for switching and augmentation, instead of reconsideration of the process in treatment selection. Fava and Rafanelli [4] have applied the concept of cascade iatrogenesis, that originated in geriatrics [44], to psychiatric settings. The patient is prescribed an increasing number of medications that, as in the STAR*D trial, in the long run cause other problems and may make the illness refractory. When symptoms of behavioral toxicity are misinterpreted or simply ignored, a cascade of events leading to illness deterioration or its chronic course may result from the choices of the clinician [4].

An initial cross-sectional examination with a very narrow focus [45] seems to generate a number of decisions that are performed in "automatic", as a result of algorithms or guidelines, with few opportunities for modifying the initial judgment.

\section{A New Look at the Problem}

Nierenberg and Amsterdam [46] introduced the term "pseudo-resistance" in depression, for indicating the presence of inappropriate dose and duration of treatment, poor adherence by the patient, or important comorbidity that may affect response. Pharmacokinetic factors, such as concomitant use of metabolic inducers, may also contribute to the phenomenon of pseudo-resistance. Another aspect of pseudo-resistance involves patients who are misdiagnosed as having unipolar depression when they suffer from diseases such as bipolar illness, vascular dementia, or anxiety disorders [46]. However, there is a more substantial question that arises. The majority of depressed patients qualify not for one, but for several psychiatric disorders [47]. Comorbid anxiety disorders were found to be the most powerful clinical factors associated with treatment-refractory depression [48]. Is a single course of an antidepressant drug an adequate treatment for a patient with complex comorbidities, having been exposed to a variety of previous treatments? 
Table 1. Staging of manifestations of oppositional tolerance in current and/or previous depressive episodes

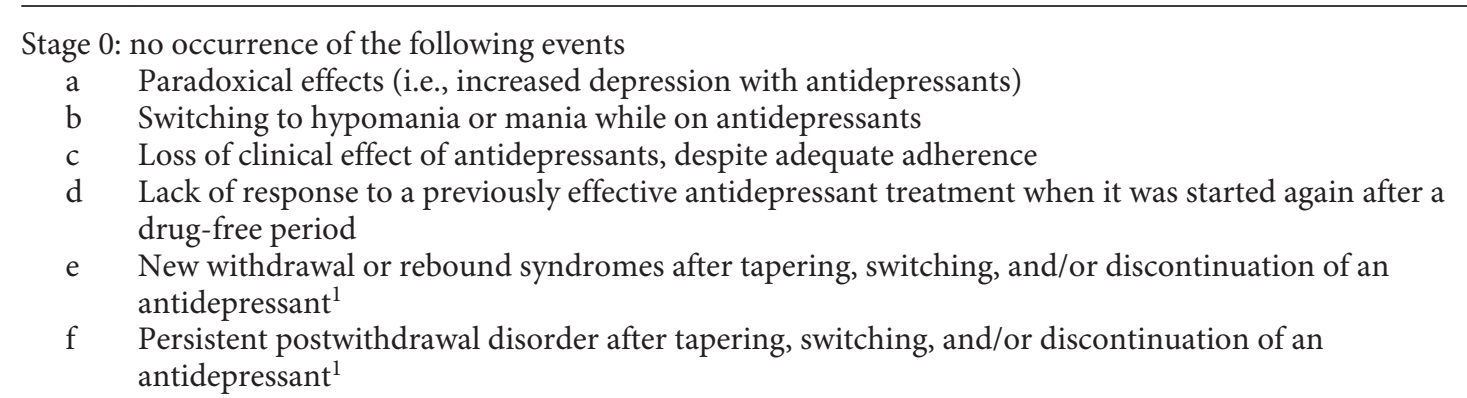

Stage 1: occurrence of 1 event

Stage 2: occurrence of 2 events

Stage 3: occurrence of 3 events

Stage 4: occurrence of 4 or more events

${ }^{1}$ According to the criteria by Cosci and Chouinard [33].

For addressing such complexities and the unsatisfactory degree of remission that current therapeutic strategies entail [19], the sequential model of treatment has been developed $[49,50]$. Such an approach has been found to entail significant benefits in terms of relapse rate [50]. The characteristic of this treatment is that sequence is performed regardless of the outcome of the first component (whether treatment failure occurred or not), as pre-planned strategy. In unipolar depression, the sequential use of pharmacotherapy and psychotherapy was found to improve long-term outcome after termination of treatment [50]. Sequential approaches do not fall within the realm of maintenance strategies and often departed from standard modalities of assessment and treatment planning. The sequential model relies on strategies such as repeated assessments, emphasis on subclinical and residual symptomatology, individualized treatment, and multidisciplinary settings [51].

Yet, psychotherapy has been largely neglected in treatment resistance in depression, despite evidence supporting its role [52, 53]. For instance, Wiles and colleagues [54] performed a RCT aimed at examining the effectiveness of cognitive-behavioral therapy (CBT) as an adjunct to usual care (including pharmacotherapy) in a large sample of primary care patients $(n=469)$ with treatmentrefractory depression compared to usual care alone. Augmenting usual care with CBT significantly increased the treatment response at 6 months compared to usual care alone (46 vs. $22 \%$ ), reducing depressive symptoms and improving quality of life in such patients. Treatment gains were maintained at a 12-month follow-up. The addition of CBT to usual care was also found to be cost-ef- fective in primary care patients who had not responded to antidepressant drugs [55], providing further support to the efficacy of CBT in this population. One may thus suggest a restrictive use of the term treatment resistance to cases who have been submitted to a sequential combination of treatments (either psychotherapy after pharmacotherapy or vice versa) to begin with. Another important aspect in arriving at a definition involves consideration of the issue of the spectrum of action of the therapeutic tool (expected responsiveness).

Once treatment resistance has been established, assessment based on diagnostic criteria and rating scales such as the Hamilton Depression Rating Scale [17] is clearly inadequate. First, using the checklist for assessing behavioral toxicity suggested by Fava and Rafanelli [4], a staging system based on the occurrence of manifestations of oppositional tolerance becomes feasible (Table 1).

Second, when Feinstein [56] introduced the concept of comorbidity, he referred to any "additional coexisting ailment" separate from the primary disease, even in the case this secondary phenomenon does not qualify as a disease per se. Indeed, in clinical medicine, the many methods that are available for measuring comorbidity are not limited to disease entities [57]. In psychiatry there is still the tendency to rely exclusively on diagnostic criteria and psychiatric symptoms, excluding other information related to, for instance, impairment, distress, and well-being $[45,58]$. The method of macro-analysis $[45,59]$ is however available and establishes a relationship between co-occurring syndromes and problems based on where treatment should begin in the first place. Macro-analysis starts from the assumption that in most cases there are 
Fig. 1. Counter-therapeutic factors contributing to treatment resistance in depression.

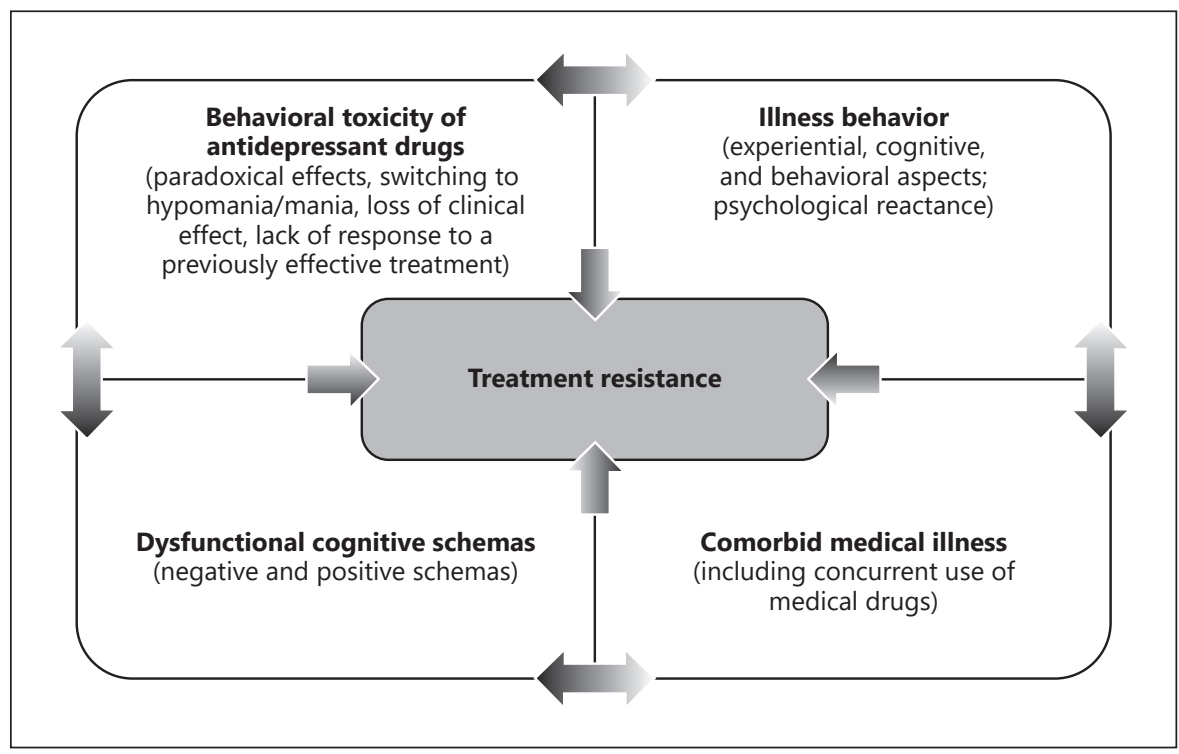

functional relationships with other more or less clearly defined problem areas, and that the targets of treatment may vary during the course of disturbances. The clinical applications of the method to the problem of treatmentresistant depression have been described in detail elsewhere [52].

Finally, treatment outcome is the cumulative result of the interaction of several classes of variables with a selected treatment: living conditions (e.g., housing, nutrition, work environment, social support), patient characteristics (e.g., age, sex, genetics, general health conditions, personality, well-being), illness features and previous therapeutic experience, self-management, and treatment setting (e.g., physician's attitude and attention, illness behavior) [42]. Such variables may be therapeutic or counter-therapeutic. In certain patients, their interactive combination may lead to clinical improvement, whereas, in other cases, it may produce no effect, and, in a third group, it may lead to worsening of the condition. Looking for counter-therapeutic ingredients is an important and yet neglected issue when treatments have failed (Fig. 1). In addition to oppositional tolerance, depression in the setting of specific medical disorders, such as Cushing's syndrome, may be associated with poor response to antidepressant drugs [60, 61]. Illness behavior, in its experiential, cognitive and behavioral aspects, is an additional important source of counter-therapeutic attitudes [62]. What the patient perceives represents the experiential aspect; the way he/she interprets such perceptions constitutes the cognitive aspect; the role of the patient in collaborating with treatment plan (self-management) con- stitutes the behavioral aspect. For instance, in psychiatric practice one can observe that certain types of patients seem to antagonize drug effects, whether this is due to psychological reactance (a motivational force that leads individuals to fear loss of control), the balance between internal and external health control beliefs, and illness behavior [63]. Such paradoxical clinical phenomena can be easily found in the setting of personality disturbances [64]. Yet they do not invariably occur in every patient with certain characteristics, because they depend on the interaction between patient and doctor.

Other sources of counter-therapeutic elements come from dysfunctional cognitive schemas: prospective studies have shown that more negatively biased cognitive schemas are associated with a worse clinical course and more severe symptomatology [65]. Conversely, the presence of unaffected areas of psychological well-being may predict a more favorable clinical course [66]. There is a wide variation in the characteristics of illness behavior in depressed patients [67] and in the response of both positive and negative schemas to treatment [66]. Both illness behavior and dysfunctional cognitive schemas may antagonize pharmacological treatment [42]. Counter-therapeutic factors are generally neglected in consideration of treatment-resistant depression, while they should be object of close clinical scrutiny. Illness behavior may be responsive to psychotherapeutic management (application of psychological understanding to the management and rehabilitation of the individual patient, including establishing a therapeutic relationship, identifying current problems with specific assessment procedures, and en- 
couraging self-therapy) which should not be confused with formal psychotherapy [68]. Yet, this is exactly what reductionistic thinking that characterizes current conceptualization of treatment resistance is likely to minimize.

\section{The Emergence of Alternative Research Designs}

A substantial weakness of the design of current RCT is the insufficient attention to previous treatments aside from response rates, with particular reference to iatrogenic comorbidity [43]. Patients are included in a trial as long as they meet certain inclusion criteria as to their current conditions. The comparison between treatment groups is thus flawed by a randomization process that does not take into account these variables [18].

An intervention can either be evaluated by a single large trial or by a series of smaller trials [69]. The standard of therapeutic trial in psychiatry is nowadays represented by the large, multicenter RCT with broad inclusion criteria (e.g., major depressive disorder), but little attention is devoted to the clinical history of patients [18]. Not surprisingly, the conclusions that can be drawn by these trials are often very limited. The idea that randomization in trials may eliminate unmeasured confounders clashes against the effects of iatrogenic comorbidity. If we peruse the literature for clinical studies concerned with samples homogeneous for treatment history, we may find out that we do not even have adequate information from observational studies or open therapeutic trials. Small trials on carefully defined populations (in terms of comorbidity subgroups and treatment history) may actually provide important clinical information that is immediately helpful to the clinician encountering that specific patient. This strategy would constitute a paradigm shift in psychiatry.

Conflicting results among randomized controlled trials can represent a spectrum of outcomes based on different patient groups more than bias or random variability [70]. If a treatment is tested by a series of small trials with inclusion criteria specific for clinical characteristics (including treatment history, subgroups, comorbidity), we may have a better knowledge of its indications and contraindications. A series of trials with differentiated recruitment strategies, such as ineffectiveness of antidepressant drugs due to loss of clinical effect [71-73] and resistance to re-challenge $[74,75]$, may provide crucial information. An issue that is often forgotten is the fact that, in front of the individual patient (who is never the average patient), the physician needs to weigh the advantages and disadvantages of each treatment option and include patient preferences [76].

\section{Conclusions}

The clinical approach to major depressive disorders, especially in the case of drug resistance or partial remission, should be filtered by clinical judgment taking into consideration a number of clinical variables such as characteristics and severity of depressive illness, co-occurring symptomatology and problems (not necessarily syndromes), medical comorbidities, and the patient's history with particular reference to previous treatments $[52,76]$. Such information should be placed within what is actually available in the specific treatment setting and should be integrated with the patient's preferences.

The ill-defined concept of treatment resistance is based on the untested assumption that treatment was right in the first place and failure to respond is shifted upon patients' characteristics. Treatment resistance thus calls for switching and augmentation which may trigger a "cascade iatrogenesis" [4] instead of reconsideration of the process in treatment selection. The naivete of this approach reminds of early research on the placebo effect and its quest for identification of the "placebo reactor," before studies identified the importance of the settings and patient-doctor interaction and demonstrated that the same subject may display a placebo response in one experimental condition and not in another [42]. Further, this approach follows a reductionistic and outdated medical model. Engel [77] outlined how the biopsychosocial model is needed to explain the number of variables operating in therapeutic encounters, such as the behavior of the physician and the relationship between the patient and the physician: "...the successful application of rational therapies is limited by the physician's ability to influence and modify the patient's behavior in directions concordant with health needs" (p. 132).

Clinical trials in psychopharmacology that neglect the issue of tolerance to previous treatments and a biopsychosocial perspective are at best useless and at worst misleading. Further, there is increasing need of expanding the content of customary clinical information, by including evaluation of variables such as stress, lifestyle, wellbeing, illness behavior, and psychological symptoms. The approach entailed by medicine-based evidence [78] for studying an individual trajectory of treatment response may unravel clinical insights that the randomized con- 
trolled trial would fail to disclose. Joint consideration of iatrogenic factors and expanded clinical information would constitute a paradigm shift in psychopharmacology and psychotherapy research in depression.

\section{Disclosure Statement}

The authors have no conflict of interest to disclose. No external funding was required for this work.

\section{References}

1 Malhi GS, Das P, Mannie Z, Irwin L. Treatment-resistant depression: problematic illness or a problem in our approach? Br J Psychiatry. 2019 Jan;214(1):1-3.

2 Demyttenaere $\mathrm{K}$. What is treatment resistance in psychiatry? A "difficult to treat" concept. World Psychiatry. 2019 Oct;18(3): $354-5$.

3 Salloum NC, Papakostas GI. Staging treatment intensity and defining resistant depression. J Clin Psychiatry. 2019 Jun;80:18r12250.

4 Fava GA, Rafanelli C. Iatrogenic Factors in Psychopathology. Psychother Psychosom. 2019;88(3):129-40.

5 Fava M. Diagnosis and definition of treatment-resistant depression. Biol Psychiatry. 2003 Apr;53(8):649-59.

6 Simpson GM, Kessel JB. Treatment-resistant depression. Br J Psychiatry. 1991 Jul;159(1): 162-3.

7 Faravelli C, Cosci F, Ciampelli M, Scarpato MA, Spiti R, Ricca V. A self-controlled, naturalistic study of selective serotonin reuptake inhibitors versus tricyclic antidepressants. Psychother Psychosom. 2003 Mar-Apr;72(2): 95-101.

8 Undurraga J, Baldessarini RJ. Direct comparison of tricyclic and serotonin-reuptake inhibitor antidepressants in randomized headto-head trials in acute major depression: systematic review and meta-analysis. J Psychopharmacol. 2017 Sep;31(9):1184-9.

9 Dold M, Bartova L, Rupprecht R, Kasper S. Dose escalation of antidepressants in unipolar depression: a meta-analysis of double-blind, randomized controlled trials. Psychother Psychosom. 2017;86(5):283-91.

10 Bschor T, Kern H, Henssler J, Baethge C. Switching the antidepressant after nonresponse in adults with major depression: a systematic literature search and meta-analysis. J Clin Psychiatry. 2018 Jan/Feb;79(1). pii: $16 r 10749$.

11 Fava GA, Savron G, Grandi S, Rafanelli C. Cognitive-behavioral management of drugresistant major depressive disorder. J Clin Psychiatry. 1997 Jun;58(6):278-82.

12 Fava GA, Kellner R. Staging: a neglected dimension in psychiatric classification. Acta Psychiatr Scand. 1993 Apr;87(4):225-30.

13 Cosci F, Fava GA. Staging of mental disorders: systematic review. Psychother Psychosom. 2013;82(1):20-34.

14 Thase ME, Rush AJ. When at first you don't succeed: sequential strategies for antidepressant nonresponders. J Clin Psychiatry. 1997; 58 Suppl 13:23-9.
15 Souery D, Amsterdam J, de Montigny C, Lecrubier Y, Montgomery S, Lipp O, et al. Treatment resistant depression: methodological overview and operational criteria. Eur Neuropsychopharmacol. 1999 Jan;9(1-2):83-91.

16 Fekadu A, Wooderson S, Donaldson C, Markopoulou K, Masterson B, Poon L, et al. A multidimensional tool to quantify treatment resistance in depression: the Maudsley staging method. J Clin Psychiatry. 2009 Feb;70(2): 177-84.

17 Carrozzino D, Patierno C, Fava GA, Guidi J. The Hamilton Rating Scales for Depression. Psychother Psychosom. 2020;89(3):133-50.

18 Fava GA, Tomba E, Tossani E. Innovative trends in the design of therapeutic trials in psychopharmacology and psychotherapy. Prog Neuropsychopharmacol Biol Psychiatry. 2013 Jan;40:306-11.

19 Fava GA, Ruini C, Belaise C. The concept of recovery in major depression. Psychol Med. 2007 Mar;37(3):307-17.

20 Horwitz RI, Singer BH, Makuch RW, Viscoli $\mathrm{CM}$. Can treatment that is helpful on average be harmful to some patients? A study of the conflicting information needs of clinical inquiry and drug regulation. J Clin Epidemiol. 1996 Apr;49(4):395-400.

21 Guidi J, Brakemeier EL, Bockting CL, Cosci F, Cuijpers P, Jarrett RB, et al. Methodological Recommendations for Trials of Psychological Interventions. Psychother Psychosom. 2018; 87(5):276-84

22 Lambert MJ. Maximizing Psychotherapy Outcome beyond Evidence-Based Medicine. Psychother Psychosom. 2017;86(2):80-9.

23 Richardson WS, Doster LM. Comorbidity and multimorbidity need to be placed in the context of a framework of risk, responsiveness, and vulnerability. J Clin Epidemiol. 2014 Mar;67(3):244-6.

24 Fava M, Martinez JM, Greist J, Marangell LB, Brown E, Chen L, et al. The efficacy and tolerability of duloxetine in the treatment of anxious versus non-anxious depression: a posthoc analysis of an open-label outpatient study. Ann Clin Psychiatry. 2007 Jul-Sep; 19(3): 187-95

25 Dubovsky SL. What Is New about New Antidepressants? Psychother Psychosom. 2018; 87(3):129-39.

26 Fava GA, Offidani E. The mechanisms of tolerance in antidepressant action. Prog Neuropsychopharmacol Biol Psychiatry. 2011 Aug; 35(7):1593-602.
27 Bosman RC, Waumans RC, Jacobs GE, Oude Voshaar RC, Muntingh AD, Batelaan NM, et al. Failure to Respond after Reinstatement of Antidepressant Medication: A Systematic Review. Psychother Psychosom. 2018;87(5): 268-75.

28 Fornaro M, Anastasia A, Novello S, Fusco A, Pariano R, De Berardis D, et al. The emergence of loss of efficacy during antidepressant drug treatment for major depressive disorder: an integrative review of evidence, mechanisms, and clinical implications. Pharmacol Res. 2019 Jan;139:494-502.

29 Chouinard G, Chouinard VA. New classification of selective serotonin reuptake inhibitor withdrawal. Psychother Psychosom. 2015; 84(2):63-71.

30 Fava GA, Belaise C. Discontinuing antidepressant drugs: lesson from a failed trial and extensive clinical experience. Psychother Psychosom. 2018;87(5):257-67.

31 Davies J, Read J. Authors' response to a critique by Jauhar and Hayes of "A systematic review into the incidence, severity and duration of antidepressant withdrawal effects: are guidelines evidence-based?" Addict Behav. 2019 Oct;97:127-30.

32 Fava GA, Cosci F. Addressing clinical challenges of antidepressant discontinuation. Am J Psychiatry. 2019;176(6):487-8.

33 Cosci F, Chouinard G. Acute and persistent withdrawal syndromes following discontinuation of psychotropic medications. Psychother Psychosom. DOI: 10.1159/000506868.

34 Fava M, Mischoulon D, Iosifescu D, Witte J, Pencina M, Flynn M, et al. A double-blind, placebo-controlled study of aripiprazole adjunctive to antidepressant therapy among depressed outpatients with inadequate response to prior antidepressant therapy (ADAPT-A Study). Psychother Psychosom. 2012;81(2): 87-97.

35 Medical Research Council Streptomycin in Tuberculosis Trials Committee. STREPTOMYCIN treatment of pulmonary tuberculosis. BMJ. 1948 Oct;2(4582):769-82.

36 Bradford Hill A. Memories of the British Streptomycin Trial in Turberculosis. Control Clin Trials. 1990 Apr;11(2):77-9.

37 Feinstein AR, Horwitz RI. Problems in the "evidence" of "evidence-based medicine". Am J Med. 1997 Dec;103(6):529-35.

38 Lavori PW, Dawson R. Adaptive treatment strategies in chronic disease. Annu Rev Med. 2008;59(1):443-53. 
39 Lei H, Nahum-Shani I, Lynch K, Oslin D, Murphy SA. A "SMART" design for building individualized treatment sequences. Annu Rev Clin Psychol. 2012;8(1):21-48.

40 Rush AJ, Trivedi MH, Wisniewski SR, Nierenberg AA, Stewart JW, Warden D, et al. Acute and longer-term outcomes in depressed outpatients requiring one or several treatment steps: a STAR*D report. Am J Psychiatry. 2006 Nov;163(11):1905-17.

41 Parloff MB, Kelman HC, Frank JD. Comfort, effectiveness, and self-awareness as criteria of improvement in psychotherapy. Am J Psychiatry. 1954 Nov; 111(5):343-52.

42 Fava GA, Guidi J, Rafanelli C, Rickels K. The clinical inadequacy of the placebo model and the development of an alternative conceptual framework. Psychother Psychosom. 2017; 86(6):332-40.

43 Fava GA, Cosci F, Offidani E, Guidi J. Behavioral Toxicity Revisited: Iatrogenic Comorbidity in Psychiatric Evaluation and Treatment. J Clin Psychopharmacol. 2016 Dec; 36(6):550-3.

44 Thornlow DK, Anderson R, Oddone E. Cascade iatrogenesis: factors leading to the development of adverse events in hospitalized older adults. Int J Nurs Stud. 2009 Nov;46(11): 1528-35.

45 Fava GA, Rafanelli C, Tomba E. The clinical process in psychiatry: a clinimetric approach. J Clin Psychiatry. 2012 Feb;73(2):177-84.

46 Nierenberg AA, Amsterdam JD. Treatmentresistant depression: definition and treatment approaches. J Clin Psychiatry. 1990 Jun;51 Suppl:39-47.

47 Zimmerman M, Chelminski I, McDermut W. Major depressive disorder and axis I diagnostic comorbidity. J Clin Psychiatry. 2002 Mar; 63(3):187-93.

48 Souery D, Oswald P, Massat I, Bailer U, Bollen J, Demyttenaere K, et al.; Group for the Study of Resistant Depression. Clinical factors associated with treatment resistance in major depressive disorder: results from a European multicenter study. J Clin Psychiatry. 2007 Jul; 68(7):1062-70

49 Fava GA, Grandi S, Zielezny M, Canestrari R, Morphy MA. Cognitive behavioral treatment of residual symptoms in primary major depressive disorder. Am J Psychiatry. 1994 Sep; 151(9):1295-9.

50 Guidi J, Tomba E, Fava GA. The Sequential Integration of Pharmacotherapy and Psychotherapy in the Treatment of Major Depressive Disorder: A Meta-Analysis of the Sequential Model and a Critical Review of the Literature. Am J Psychiatry. 2016 Feb;173(2):128-37.
51 Guidi J, Tomba E, Cosci F, Park SK, Fava GA. The role of staging in planning psychotherapeutic interventions in depression. J Clin Psychiatry. 2017 Apr;78(4):456-63.

52 Guidi J, Fava GA. Psychosocial management of treatment resistant mood disorders: current evidence. In: Carvalho AF, McIntyre RS, editors. Treatment resistant mood disorders. Oxford: Oxford University Press; 2015. p. 95105.

53 van Bronswijk S, Moopen N, Beijers L, Ruhe HG, Peeters F. Effectiveness of psychotherapy for treatment-resistant depression: a metaanalysis and meta-regression. Psychol Med. 2019 Feb;49(3):366-79.

54 Wiles N, Thomas L, Abel A, Ridgway N, Turner N, Campbell J, et al. Cognitive behavioural therapy as an adjunct to pharmacotherapy for primary care based patients with treatment resistant depression: results of the CoBalT randomised controlled trial. Lancet. 2013 Feb;381(9864):375-84.

55 Hollinghurst S, Carroll FE, Abel A, Campbell J, Garland A, Jerrom B, et al. Cost-effectiveness of cognitive-behavioural therapy as an adjunct to pharmacotherapy for treatmentresistant depression in primary care: economic evaluation of the CoBalT Trial. Br J Psychiatry. 2014 Jan;204(1):69-76.

56 Feinstein AR. The pre-therapeutic classification of comorbidity in chronic disease. J Chronic Dis. 1970 Dec;23(7):455-68.

57 de Groot V, Beckerman H, Lankhorst GJ, Bouter LM. How to measure comorbidity. a critical review of available methods. J Clin Epidemiol. 2003 Mar;56(3):221-9.

58 Feinstein AR. Clinimetrics. New Haven (CT): Yale University Press; 1987. https://doi. org/10.2307/j.ctt1xp3vbc.

59 Emmelkamp PM, Bouman T, Scholing A. Anxiety disorders. Chichester: Wiley; 1993.

60 Fava GA, Sonino N. Depression associated with medical illness. CNS Drugs. 1996;5(3): 175-89.

61 Cosci F, Fava GA, Sonino N. Mood and anxiety disorders as early manifestations of medical illness: a systematic review. Psychother Psychosom. 2015;84(1):22-9.

62 Cosci F, Fava GA. The clinical inadequacy of the DSM-5 classification of somatic symptom and related disorders: an alternative trans-diagnostic model. CNS Spectr. 2016 Aug;21(4): $310-7$.

63 de las Cuevas C, de Leon J. Reviving research on medication attitudes for improving pharmacotherapy: focusing on adherence. Psychother Psychosom. 2017;86(2):73-9.
64 DiMascio A. Personality and variability of response to psychotropic drugs: relationship to "paradoxical" effects. In: Rickels K, editor. Non specific factors in drug therapy. Springfield (IL): Charles C. Thomas; 1968. pp. 40-9.

65 LeMoult J, Gotlib IH. Depression: a cognitive perspective. Clin Psychol Rev. 2019 Apr;69: 51-66.

66 Fava GA, Guidi J. The pursuit of euthymia. World Psychiatry. 2020 Feb;19(1):40-50.

67 Guidi J, Fava GA, Picardi A, Porcelli P, Bellomo A, Grandi S, et al. Subtyping depression in the medically ill by cluster analysis. J Affect Disord. 2011 Aug;132(3):383-8.

68 Fava GA. Rational use of antidepressant drugs. Psychother Psychosom. 2014;83(4): 197-204.

69 Borm GF, Donders R. A treatment should be evaluated by small trials. J Clin Epidemiol. 2009 Aug;62(8):887-9.

70 Jane-wit D, Horwitz RI, Concato J. Variation in results from randomized, controlled trials: stochastic or systematic? J Clin Epidemiol. 2010 Jan;63(1):56-63.

71 Schmidt ME, Fava M, Zhang S, Gonzales J, Raute NJ, Judge R. Treatment approaches to major depressive disorder relapse. Part 1: dose increase. Psychother Psychosom. 2002 Jul-Aug;71(4):190-4.

72 Fava GA, Ruini C, Rafanelli C, Grandi S. Cognitive behavior approach to loss of clinical effect during long-term antidepressant treatment: a pilot study. Am J Psychiatry. 2002 Dec;159(12):2094-5.

73 Fabbri S, Fava GA, Rafanelli C, Tomba E. Family intervention approach to loss of clinical effect during long-term antidepressant treatment: a pilot study. J Clin Psychiatry. 2007 Sep;68(9):1348-51.

74 Fava M, Schmidt ME, Zhang S, Gonzales J, Raute NJ, Judge R. Treatment approaches to major depressive disorder relapse. Part 2: reinitiation of antidepressant treatment. Psychother Psychosom. 2002 Jul-Aug;71(4): 195-9.

75 Franchini L, Rossini D, Bongiorno F, Spagnolo C, Smeraldi E, Zanardi R. Will a second prophylactic treatment with a higher dosage of the same antidepressant either prevent or delay new depressive episodes? Psychiatry Res. 2000 Sep;96(1):81-5.

76 Tomba E, Fava GA. Treatment selection in depression: the role of clinical judgment. Psychiatr Clin North Am. 2012 Mar;35(1):87-98.

77 Engel GL. The need for a new medical model: a challenge for biomedicine. Science. 1977 Apr;196(4286):129-36.

78 Lobitz G, Armstrong K, Concato J, Singer BH, Horwitz RI. The biological and biographical basis of precision medicine. Psychother Psychosom. 2019;88(6):333-40. 